\title{
Le pragmatisme, Mead et le tournant pratique
}

Pragmatism, Mead and the Practice Turn

\section{Barbara Simpson}

Traducteur : Christian Brassac

\section{OpenEdition \\ Journals}

Édition électronique

URL : http://journals.openedition.org/activites/622

DOI : $10.4000 /$ activites. 622

ISSN : 1765-2723

Éditeur

ARPACT - Association Recherches et Pratiques sur les ACTivités

Référence électronique

Barbara Simpson, « Le pragmatisme, Mead et le tournant pratique », Activités [En ligne], 10-1 | Avril 2013, mis en ligne le 15 avril 2013, consulté le 20 avril 2019. URL : http://journals.openedition.org/ activites/622 ; DOI : 10.4000/activites.622

\section{(c) (i) (2) $\Theta$}

Activités est mis à disposition selon les termes de la licence Creative Commons Attribution - Pas d'Utilisation Commerciale - Pas de Modification 4.0 International. 


\title{
Le pragmatisme, Mead et le tournant pratique
}

\section{Barbara Simpson}

\author{
Strathclyde Business Scholl, Department of Management, 199 Cathedral Street, G4 OQU Glasgow (UK) \\ barbara.simpson@strath.ac.uk \\ traduction Christian Brassac
}

\begin{abstract}
Pragmatism, Mead and the Practice Turn. 'Practice' is a familiar term in everyday language but it also has a long history of scholarship. What then does it mean to 'turn' to wards practice, and how would we know when a practice turn has occurred? To answer these questions, this article develops a theoretical view of practice as a transactional social process involving experience and action as mutually informing aspects of human conduct. This perspective is elaborated in detail by drawing on the ideas of the pragmatist philosophers, especially George Herbert Mead. In particular, it is asserted that 'transactionality' and 'temporality', when taken together, offer a theoretical perspective on practice that is dynamic, emergent and socially agentic. The utility of this pragmatist approach is illustrated using a published study of astrategizing episode. The article concludes that a practice turn is indeed underway in organization studies, but there is still some distance to travel before the full potential of this turn is realized.
\end{abstract}

\section{KEYWORDS}

emergence, pragmatism, social agency, temporality

Les chercheurs en sciences des organisations se sont toujours intéressés à ce que les gens font effectivement, mais c'est seulement dans la dernière décennie ou à peu près que le thème de la pratique a pris son essor. Cette tendance est tout à fait évidente dans les sousdomaines de la stratégie comme pratique (Jarzabkowski, 2007 ; Johnson, Balogun, \& Seidl, 2003) et de l'apprentissage organisationnel (Nicolini, Gherardi, \& Yanow, 2003), où les dynamiques de la pratique humaine sont toutes aussi importantes que les résultats qu !ils produisent. Whittington $(2004,2006)$ a avancé que ce mouvement reflète un 'tournant pratique' ('practice turn') qui est apparu en réponse aux nombreuses frustrations dues à la déconnection entre la théorisation académique et les expériences pratiques de l'organisation (dans le double sens de processus d'organisation et de son résultat, l'organisation). Mais à quoi renvoie-t-on effectivement quand on parle d'un 'tournant' et comment peut-on vraiment savoir quand le 'tournant' a eu lieu ?

Dans une théorie, on parle usuellement d'un tournant (tels par exemple le tournant cognitif ou le tournant linguistique) lorsqu'un mouvement de pensée s'engage dans un nouveau domaine ontologique et épistémologique (Chia \& MacKay, 2007). Kuhn (1975) appelle changement de paradigme ce type de mouvement, comme lorsque, par exemple en science,

1 Je note systématiquement en italiques et entre parenthèses le mot anglais du texte quand je le juge utile pour une meilleure compréhension du texte original. Je suis à la lettre l'usage par l'auteur des ' '. 
la mécanique newtonienne fut supplantée par la vision einsteinienne de l'univers. Il est cependant important de noter qu'un tel tournant n'est pas simplement une remise en cause d'une 'science normale'; il ouvre également de nouvelles frontières intellectuelles, invite à de nouvelles façons de voir et soulève de nouvelles questions à traiter. Il requiert une contestation rigoureuse des idées et un examen approfondi des principes philosophiques qui façonnent nos discours conceptuels. Je voudrais suggérer qu'un tel débat est un signe de bonne santé d'un 'tournant en progression'.

Dans les sciences des organisations, les débats émergents sur la pratique ont été caractérisés par la recherche de théories appropriées et par le travail de théoriciens capables d'informer de nouvelles perspectives. Ainsi, par exemple, de nouvelles et intéressantes idées ont été tirées de la pensée de Giddens (Jarzabkowski, 2004 ; Whittington, 2006), de Bourdieu et Heidegger (Chia \& Holt, 2006), de Vygotsky (Jarzabkowski, 2003 ; Miettinen, 1999), de Wittgenstein (Shotter, 2006), de Foucault (Ezzamel \& Willmott, 2004) et des études relatives aux sciences et des technologies (Pickering, 1995; Orlikowski, 2000). Dans cet article, mon objectif est d'ajouter une autre voix à ces débats, la voix de la philosophie pragmatiste. Je défends l'idée selon laquelle cette perspective apporte de nouveaux éléments qui risquent d'être oubliés dans les débats tels qu'ils sont conduits actuellement. Elle offre en particulier un moyen de réunir les aspects routinier et créatif de la pratique tout en transcendant la séparation problématique entre les niveaux individuel et social des analyses.

Il s'agit d'un article théorique dont le but est de suivre à la trace le progrès du tournant vers la pratique dans le domaine des sciences des organisations. Mon enquête cherche en particulier à comprendre comment la perspective pragmatiste sur la pratique pourrait conforter ce progrès en apportant de nouvelles façons de voir et de nouvelles questions à poser. La section suivante propose une brève et nécessairement sélective revue des développements de la théorie de la pratique, identifiant les dimensions créative et agentique (agentic) de la pratique comme des domaines qui requièrent des développements théoriques supplémentaires. Je poursuivrai en proposant une vision de la pratique que l'on peut tirer des idées des pragmatistes américains et spécialement de celles de George Herbert Mead. La figure saillante de cette vision pragmatiste est son approche holistique de la pratique, approche permettant de mettre en question la dominance des théories de l' 'action rationnelle' et de l' 'action orientée par la norme' qui marque le débat contemporain sur la pratique (Joas, 1996). Le pragmatisme est cependant de peu d'intérêt sans application pratique; aussi continuerai-je en examinant une étude empirique, relevant du sous-domaine de la stratégie et déjà publiée, me permettant mettre à jour de nouvelles idées issues du point de vue pragmatiste sur la pratique. Pour finir, je discute les potentialités de cette vision pragmatiste d'améliorer les conceptions actuelles de la pratique et de donner un nouvel élan au tournant pratique.

\section{La pratique en théorie}

Dans l'usage actuel, le mot 'pratique' est devenu un terme ambigu que Bernstein (1972) caractérise comme détenant des interprétations 'basse' et 'haute'. D'un côté nous avons affaire aux faisabilités pratiques nécessaires pour pouvoir suivre le tourbillon des choses dans un monde dynamique et incertain, champ dans lequel il y a peu de place pour la théorisation ; d'un autre côté, nous avons affaire aux activités, informées philosophiquement et théoriquement, constituant la praxis d'une vie sociale réactive et responsable. Bernstein (1972) a fait remonter le développement de la théorie de la pratique à des travaux philosophiques de la fin du dix-neuvième siècle et du début du vingtième. Il met en avant en particulier les efforts dont l'objectif est d'aller au-delà des limites du principe dialectique hégélien, qui considère l'action humaine comme un déploiement de potentialités sur fond de doutes et de luttes vers une auto-réalisation et vers la sagesse d'une vie bien vécue. Bernstein considère en particulier la théorie sociale marxiste de la pratique, l'approche très différente 
des philosophes existentialistes (Kierkegaard et Sartre) qui voient l'action en termes de conscience et d'existence, les pragmatistes (Dewey et Peirce) dont la préoccupation centrale est de développer une philosophie de l'action pratique, et finalement les philosophes analytiques qui travaillent sur la notion wittgensteinienne du discours vu comme forme d'action. En dépit de profondes différences entre leurs hypothèses, leurs idées saillantes et leurs approches, ces quatre courants intellectuels se sont tous néanmoins colletés avec les mêmes expériences de vie dans le monde de tous les jours.

Joas (1996) soutient que la recherche d'une théorie adéquate de l'action humaine ne relève en aucune façon de la seule compétence des philosophes. D'une pertinence particulière pour les sciences des organisations, les domaines de la sociologie, de la psychologie, de l'économie ont chacun développé des perspectives théoriques sur l'action humaine, mais rarement en croisant leurs travaux. Dans une exploration critique de grande ampleur, Joas a méticuleusement décortiqué les théories classiques de l'action pour mettre à jour un dualisme sous-jacent qui traverse nos compréhensions contemporaines de la pratique. Les théories de 1' 'action rationnelle', telles celles qui caractérisent la discipline de l'économie, suggèrent une orientation volontariste dans laquelle l'acteur individuel exerce sa liberté de choix, alors que les sociologues tendent à insister sur le déterminisme structurel dans les théories de l'action 'normativement orientées'. Cette ligne de partage théorique est certainement sous-tendue par des questions d'agentivité (agency) et de liberté d'agir, mais du point de vue de Joas, aucune de ces positions théoriques ne rend compte adéquatement de la dimension créative de l'agentivité humaine.

L'état actuel de la théorie de la pratique est également évalué par Schatzki (2001) dans sa revue critique du tournant pratique en théorie sociale. Il recense trois catégories de travaux. Il identifie premièrement un ensemble de théories qui trouvent leurs racines dans des travaux philosophiques et sociologiques relatifs à l'établissement et au maintien de structures sociales stables et durables (voir également Reckwitz, 2002). La mise en avant du social est devenue particulièrement évidente dans les écrits post-structuralistes qui, sous l'influence de Nietzsche et Heidegger, ont adopté une attitude profondément sceptique vis-à-vis de la capacité humaine à l'action agentique. La dynamique de cet ensemble se traduit par une convergence vers l'équilibre. Dans les sciences des organisations, cette dynamique se reflète dans les réflexions récentes à propos de, par exemple, la vision culturaliste de l'apprentissage organisationnel (Cook \& Yanow, 1993 ; Yanow, 2000), les communautés de pratiques (Lave \& Wenger, 1991 ; Wenger, 2000), et la durabilité des identités socialement construites (Kärreman \& Alvesson, 2001). Les travaux de la seconde catégorie identifiée par Schatzki sont également dans une dynamique convergeant vers une micro-perspective sur les activités humaines. Le focus est plutôt mis sur les dimensions psychologiques associées à l'incarnation de la signification et sur les dispositions et compréhensions implicites qui, à la fois, modèlent et sont modelées par la pratique. De façon surprenante, cette perspective apparaît comme étant moins bien représentée dans la littérature sur les organisations bien que ces travaux fassent preuve d'efforts significatifs pour être au plus près des activités des individus en temps réel. Par exemple, Johnson et al. (2003, p. 3) travaillent à une plus approfondie « compréhension de la myriade des micro-activités » de la pratique à la manière de ce qui est réalisé par Samra-Fredericks $(2003,2004)$ dans son travail sur le discours en temps réel.

Schatzki range dans la troisième catégorie ces théories de la pratique qui sont inspirées par les auteurs post-humanistes, appartenant plus spécialement aux études des sciences et technologies (science and technology studies) (Callon, 1986 ; Harvey \& Haraway, 1985 ; Knorr Cetina, 2001 ; Latour, 1987 ; Pickering, 1995). Contrairement aux deux premières catégories, celle-ci est caractérisée par une dynamique divergente d'émergence et de transformation qui résonne avec le souhait de Joas de voir se développer une théorisation adaptée à la dimension créative de l'action. Les exemples de la littérature sur les organisations où cette approche a été utilisée sont les applications de la théorie de l'activité 
(Blackler, Norman, McDonald, \& Seonaidh, 2000 ; Miettinen, 1999) et les études sur les objets vus comme médiateurs dans les processus de construction de sens (Engeström, Puonti, \& Seppänen, 2003 ; Orlikowski, 2000 ; Simpson \& Caroll, 2008). Bien que, sans aucun doute, ces études s'intéressent à la dynamique des processus et à la construction créative de significations, elles le font souvent d'une manière quelque peu désincarnée, tendant à dévaluer l'expérience subjective de l'agentivité.

Ces différentes approches de la théorisation de la pratique mettent l'accent sur deux thématiques primordiales. Premièrement, la pratique est un processus dynamique, temporel qui converge et diverge à la fois. C'est en ce sens où la pratique peut tirer des idées de la littérature sur le processus (Jarzabkowski, 2004 ; Linstead, 2002 ; Rescher, 2000 ; Tsoukas and Chia, 2002). La pratique est cependant plus qu'un simple processus temporel; elle implique nécessairement la conduite humaine et l'exercice de l'agentivité. En cela, je ne veux pas seulement parler de l'agentivité auto-centrée, intentionnelle et excessivement psychologisée qui a été largement critiquée dans les années récentes. Je veux plutôt suggérer une forme d'agentivité socialement incarnée qui transcende les frontières traditionnelles entre individu et social (voir également Emirbayer \& Mische, 1998). Un défi majeur pour le progrès du tournant pratique est par conséquent de développer de nouvelles perspectives qui s'attachent à l'agentivité à la fois sociale et temporelle.

La discussion conduite jusqu'ici fait bien apparaitre que la théorie de la pratique est habitée par des dualismes: théorie versus pratique, 'basses' versus 'hautes' pratiques, action rationnelle versus action normativement orienté, dynamique convergente versus divergente, niveaux d'analyse individuel versus social, et la liste n'est pas close. Joas (1996) soutient qu'une théorie compréhensive de la pratique ne peut être fondée sur ces dualismes, car l'action humaine, bien comprise, transcende toutes ces frontières que ces dualismes imposent à notre pensée. Il suggère en fait que ces dualismes constituent d'importants points faibles dans les sciences sociales contemporaines (voir aussi Khnights \& Mueller, 2004 ; Kilpinen, 1998). Cette question a bien été identifiée dans la théorie de la structuration de Giddens et dans la notion d'habitus de Bourdieu, les deux critiquant la séparation entre l'individuel et le social. Dans les deux cas, c'est la pratique qui rapproche ces opposés dualistiques, mais ni la structuration ni l'habitus n'engagent réellement l'action socialement incarnée sans laquelle la pratique n'a pas de support.

On trouve également des exemples de chercheurs qui s'attaquent à cette question des dualismes dans la théorie de la pratique dans les sciences des organisations. Ainsi, par exemple, Whittington (2006) prend en charge la question de ce qu'il a décrit comme une bifurcation dans la littérature sur la stratégie séparant les activités intra-organisationnelles et extra-organisationnelles, alors que Jarzabkowski (2004) s'intéresse à la tension qu'elle observe entre les tendances récursive et adaptative dans le management stratégique. Ces deux auteurs placent leur argumentation dans les débats contemporains relatifs à la théorie de la pratique tout en trouvant de nouvelles voies pour dépasser les difficultés associées à ces dualismes. Whittington construit un modèle fondé sur trois notions clés, les Pratiques, la Praxis et les Praticants (Practitionners), alors que Jarzabkowski propose que les 'pratiquesen-usage' ('practices-in-use') pourraient être les unités d'analyse appropriées dans les études empiriques. Cependant, dans le contexte des nouvelles propositions faites, aucun article ne re-théorise sérieusement l'agentivité et, ainsi, les 'comments' et 'pourquois' de la pratique restent sous-explorés. Sans cette re-théorisation compréhensive et délibérée, il est inévitable que les notions de conduite humaine et d'agentivité prévalant dans la 'science normale' ('action rationnelle' et 'action normativement orientée') n'entravent pas les progrès vers le plein achèvement du tournant pratique dans les sciences des organisations.

Par conséquent, ma conclusion est que persistent toujours des manques significatifs dans les théorisations de la pratique, particulièrement à propos des questions du 'comment' les pratiques se déploient. Pour développer une recherche en ce sens, j'ai choisi de suivre la voie 
proposée par Bernstein et Joas. Cette philosophie est caractérisée par une opposition franche aux dualismes et apporte des moyens pour résoudre ce problème clé dans les théories courantes de la pratique. Dans la section suivante, je vais dégager les aspects de la pensée pragmatiste qui concernent précisément la pratique, en accordant une attention particulière aux agentivité et temporalité sociales qui, dans cette brève revue, sont apparues comme des questions clés pour la progression du tournant pratique.

\section{La pratique à partir d'une perspective pragmatiste}

Le pragmatisme américain naît entre le milieu du dix-neuvième siècle et celui vingtième et est essentiellement le fait des écrits de Charles Sanders Peirce, William James, John Dewey et George Herbert Mead. Leur engagement commun fut de développer une philosophie des sciences qui soit applicable à, et informée par, l'expérience humaine et à la pratique. La plupart de leurs idées tournent autour des hypothèses selon lesquelles nous sommes tous des participants (pratiquants (practitioners)) dans nos mondes sociaux. C'est à travers notre participation que nous construisons et re-construisons continûment les significations sociales qui modèlent nos pensées et nos actions. Les thèmes clés de cette pensée pragmatiste originale n'ont rien perdu de leur force dans le climat intellectuel actuel. Ces idées ont d'ailleurs récemment bénéficié d'un certain regain d'intérêt, néo-pragmatiste, en ce sens où leur pertinence dans les débats transdisciplinaires contemporains et post-modernes est de plus en plus reconnue (Aboulafia, 1999; Bernstein, 1972 ; Burkitt, 1993 ; Cook, 1993 ; Dunn, 1997 ; Joas, 1997 ; Emirbayer \& Mische, 1998 ; Powell, 2002 ; Rorty, 1999).

Dans cet article j'ai choisi Mead pour principal informateur. Il a travaillé de façon très étroite avec John Dewey tout au long de sa vie professionnelle et l'on trouve de nombreux parallèles entre leurs trajectoires intellectuelles. La contribution spécifique de Mead tient cependant dans la précision et le détail analytique qu'il apporte pour compléter le programme de recherche pragmatiste plus large de Dewey (Morris, 1934). Ses descriptions empiriques, spécialement celles qui sont liées à l'action humaine, vont bien au-delà des limites usuelles de la philosophie. Un avertissement important doit cependant être fait avant de discuter ces idées. Mead est souvent associé à l'interactionnisme symbolique qui s'est développé dans les décennies qui ont suivi sa mort en 1931, mais ce mouvement s'est approprié ses idées de façon partiale et fragmentée. Blumer $(1969$, p. 1) le confirme quand il dit qu'il «s'appuie sur la pensée de George Herbert Mead qui, plus que tout autre, constitue le fondement de l'interactionnisme symbolique, mais [qu'il] s'est imposé de développer [sa] propre version ». Ce faisant, Blumer a perdu de vue les bases radicales de la philosophie de Mead qui commencent seulement maintenant à refaire surface dans les débats actuels. Aussi, mon opinion est-elle que l'on comprend mieux les idées de Mead à travers une confrontation directe avec son œuvre considérable (plus de cent articles et documents) plutôt qu'à travers le recours à des connections posthumes à l'interactionnisme symbolique.

Les efforts intellectuels de Mead sont dirigés vers le développement d'une théorie de la socialité intégrant les processus dynamiques, l'émergence et l'évolution. Son hypothèse fondamentale est que 1' 'acte social' constitue la base de toute construction de signification humaine. À travers nos actions et engagements sociaux, nous ne faisons pas que renforcer les éléments de la structure sociale que nous partageons, mais nous explorons et reconstruisons de façon créative les significations. Dans le but de capturer ces processus dans toute leur complexité, Mead s'est rendu compte de la nécessité de placer les actions sociales dans le flux temporel. Sa quête d'une théorie temporellement intégrée de la socialité reste sa contribution la plus importante à une théorie dynamique de l'action sociale. Dans la suite, je vais donner les détails de son argumentation relative à la pratique en utilisant deux thématiques phares: la transactionalité (Mead, 1925; Mead, 1934, spécialement les chapitres 3, 9, 21, 22 et 33) et la temporalité (Mead, 1932; Mead, 1938). Bien que la séparation de ces deux thématiques constitue un dispositif commode pour le développement 
de mon argument, la transactionalité et la temporalité doivent être envisagées comme des aspects étroitement intriqués et inséparables dans la théorie meadienne de la socialité.

\section{Transactionalité}

Selon Mead les significations sociales sont construites à travers nos actions sociales. Sa célèbre définition de 1' 'acte social' comme conversation par gestes (conversation of gestures) stipule que le geste d'une personne appelle une réponse chez une autre personne qui, en retour, appelle une autre réponse et ainsi de suite. Ces conversations par gestes constituent le lieu où les significations sociales sont construites, renforcées et perturbées, et composent, dans le même temps, les moyens par lesquels nous venons à nous comprendre les uns les autres, et nous-mêmes, en tant que nous sommes mutuellement et socialement constitués. Bien que Mead lui-même utilise le terme 'interaction' pour décrire cette conversation par gestes, cela crée une confusion potentielle avec les théories 'interactionnistes'. C'est la raison pour laquelle Dewey et Bentley (1949[1991]) font une distinction entre inter-actions et trans-actions qu'ils voient comme deux niveaux distincts d'analyse. Ils définissent cette distinction de niveaux sur la base de huit dimensions différentes, fournissant ainsi une compréhension richement élaborée de leurs différences (Dewey \& Bentley, 1949[1991], pp. 113-115). Pour les besoins de cet article, il est suffisant de dire que là où l'inter-action est quelque chose qui se passe entre des acteurs qui sont physiquement et mentalement indépendants, une trans-action se réalise entre des acteurs qui sont des aspects d'un tout relationnellement intégré; que là où les significations sont transmises entre des acteurs en inter-action, les acteurs sont la signification continûment émergente de la trans-action. Ce concept de 'transaction' est entièrement cohérent avec l'idée de la 'conversation par gestes' de Mead. En conséquence, j'utiliserai 'transaction' de préférence au moins précis 'interaction' pour le reste de mon argument dans ce texte.

Le cycle transactionnel de gestes et de réponses est, bien sûr, rendu possible par le langage, langage que Mead ne considère pas seulement comme étant verbal, mais comme constitué d'une multiplicité de signes et symboles qui façonnent les significations sociales. Il met en avant en particulier les 'symboles signifiants' ('significants symbols'), qui sont ces gestes qui appellent la même réponse chez celui qui fait le geste que chez celui qui y répond. Ces symboles deviennent signifiants au sens où ils possèdent des significations mutuellement acceptées. Par exemple, si vous et moi avions une conversation à propos de la nature du travail académique, on devrait faire que les notions telles que 'étudiant', 'ordinateur' et 'citation' deviennent des symboles signifiants dans nos efforts de co-construction de significations. Ces symboles signifiants médiatisent nos processus transactionnels de construction de significations en identifiant des interprétations que nous tenons comme plus ou moins communes. Inversement, en l'absence de tels symboles signifiants, notre conversation se réduirait à une série de réactions réflexes qui ne pourrait pas produire de nouvelles significations. L'engagement transactionnel offre l'opportunité aux acteurs d'explorer les différences dans les significations qu'ils attachent aux symboles particuliers. Ce sont ces ambiguïtés qui rendent possibles de nouvelles idées et l'apprentissage.

Les symboles signifiants sont l'essence de la socialité en ce sens qu'ils permettent de prendre la place de l'autre au cours de la transaction et d'anticiper les réponses probables à nos propres gestes. Il est important de dire que leur fonction n'est pas seulement de médiatiser la construction transactionnelle de significations, mais également de modérer la conduite sociale. Par exemple, lorsque je fais le geste de me lever devant une classe, les membres de la classe et moi-même comprennent que le cours est sur le point de commencer et, ainsi, nous nous conduisons tous en conséquence. Cependant cela ne signifie pas nécessairement que tous les membres de la classe vont s'asseoir et être attentifs, car bien qu'un symbole signifiant indique la conduite qui peut être anticipée dans une situation sociale donnée, les individus font au final leurs propres choix pour agir. Néanmoins même ces choix sont 
socialement modérés par les symboles signifiants. Mead explique cette régulation sociale de la conduite en termes d' 'autrui généralisé' ('generalized other') qui est le système organisé de symboles signifiants qui reflète les attitudes généralisées, ou le discours, d'un groupe social ou d'une communauté. Les habitudes sociales de conduite sont constituées comme l'autrui généralisé et l'appartenance à une communauté est démontrée par une capacité à se conduire selon les attitudes généralisées. Il ne serait pas possible de s'engager dans des activités coordonnées complexes sans un tel système généralisé de symboles signifiants.

L'autrui généralisé peut également être un acteur dans des conversations transactionnelles. Ainsi par exemple, quand je téléphone à ma banque, je parle toujours avec quelqu'un que je ne connais pas (en fait quelqu'un qui est probablement dans un centre d'appel dans un autre pays), mais de mon point de vue je suis en train de mener une transaction avec 'la banque' et ma construction de significations est médiatisée par mon appréhension de 'la banque' comme un système généralisé de symboles signifiants. La personne à laquelle je parle est la voix de 'la banque' et j'anticipe sa conduite comme étant fondée sur les transactions passées que j'ai eues avec les banques. En ce sens la conversation par gestes de Mead rend possible des transactions extra-personnelles, comprenant des transactions impliquant deux, ou plus, autruis généralisés comme quand deux cultures organisationnelles entrent en conflit. Le point commun entre tous ces différents types de transactions est que les significations sont continûment construites et reconstruites à travers ces processus.

J'ai jusqu'à présent suggéré que les transactions peuvent impliquer des individus spécifiques ou des autruis généralisés, mais on peut dire qu'elles peuvent également être intrapersonnelles. Mead explique ceci en évoquant deux aspects mutuellement constitutifs du soi (self) : le 'moi' ('me') objectif et le 'je' (' $I$ ') subjectif. Le 'moi' est l'ensemble organisé des attitudes des autres, incarnées dans les symboles signifiants. Ce 'moi' incarné équivaut à des habitudes de conduite qui ont été acquises réflexivement au cours des transactions. C'est cet aspect du soi qui est accessible à la conscience, à l'examen réflexif. Dès que le 'moi' commence à naître, l'acteur est capable d'adopter une attitude réflexive envers le soi, converser avec le soi et produire transactionnellement des significations de la même manière qu'avec les autres. Le 'je' subjectif, d'un autre côté, est constitué par les réponses anticipées de l'acteur vis-à-vis des conventions sociales et des habitudes de conduite représentées par le 'moi' ; ainsi le 'je' à la fois en appelle à et répond au 'moi' dans une conversation par gestes internalisée. Mead soutient que le 'moi' et le 'je' sont sociaux de façon inhérente et sont des ressources mutuellement informées dans la construction des soi sociaux. Sans le principe du 'je', le soi ne serait rien d'autre qu'un reflet stable et convergent de la structure sociale et il n'y aurait pas de possibilités d'une activité créatrice et reconstructrice.

Pour résumer cette partie de mon argumentation, on peut dire que l'action sociale est comprise en termes de construction transactionnelle de significations, les transactions apparaissant en temps réel et à tous les niveaux du système social en commençant par le niveau intra-personnel. Dans tous les cas, les acteurs sont les significations qui émergent dans les transactions et ils exercent une forme d'agentivité en modelant ces significations. Du fait que cette agentivité est à la fois médiatisée et modérée par les symboles signifiants, elle possède une qualité sociale inhérente. Ceci me conduit à une définition de l'agentivité sociale qui est une des deux dimensions clé de la pratique que j'ai identifiée dans ma précédente revue la littérature de la pratique. L'agentivité sociale est la capacité d'influencer les significations des actions sociales. Les transactions sont les lieux où s'exerce l'agentivité sociale et, puisque les transactions sont médiatisées par les symboles signifiants, l'agentivité sociale ne peut jamais être attribuée à un acteur singulier. En d'autres termes, un geste n'a pas de capacité agentique (agentic capacity) indépendamment du fait qu'il fasse appel à un type donné de réponse. 


\section{Temporalité}

Mead soutient que les transactions ne peuvent à elles seules fournir une formulation adéquate de la sociabilité humaine. L' 'acte social' est nécessairement également temporel. Il considère en particulier que la socialité est plus qu'une simple succession de moments transactionnels ; elle implique aussi la narration continue des soi sociaux en devenir. Mead fut en la matière influencé par Henri Bergson (1919) qui faisait une distinction importante entre la spatialisation du temps, moment par moment, et le flux continu de la durée (voir aussi Linstead, 2002 ; Tsoukas \& Chia, 2002). Bergson soutenait que la signification du temps repose sur l'expérience introspective de la durée, alors que le temps spatialisé est une simple distorsion de la temporalité authentique. Bien que Mead soit certainement d'accord avec la nécessité de rejeter la conception classique et dogmatique du temps spatialisé, il ne suit pas l'option de Bergson privilégiant l'expérience introspective (Mead 1963-4). Il conçoit plutôt les événements objectifs et les expériences subjectives de la continuité comme étant fortement intriqués et synthétisés dans la conduite humaine. Il soutient que les événements objectifs sont essentiels à la structuration du flux temporel, flux temporel qui ne peut être expériencé que lorsqu'il est interrompu par la survenance d'un événement qui pousse vers la création de nouvelles possibilités émergentes. En ce sens, la pensée de Mead diffère significativement de celle des philosophes du processus dont les théorisations portent prioritairement sur la dynamique du flux et sur le mouvement (Rescher, 2000 ; Whitehead, 1920).

On trouve une présentation détaillée de la pensée meadienne à propos de la dynamique de l'action humaine dans Philosophy of the Act (Mead, 1938). Son point de départ est que nous sommes toujours engagés dans un certain type d'action, dont une grande partie est pilotée par les habitudes de conduite que nous avons déjà importées dans le 'moi'. La pensée réflexive, qui est considérée comme un processus fondamentalement social, peut être stimulée lorsqu'un quelconque obstacle arrête ou inhibe ce flux d'action. L'objectif est alors de trouver un moyen pour poursuivre cette activité d'une façon ou d'une autre. Il est possible de résoudre ces situations au moyen de simples réactions réflexes qui n'impliquent par la pensée. Mead, tout comme Dewey, est cependant plus intéressé par le processus analytique de délibération, la pensée réflexive, qu'il modélise selon les quatre étapes suivantes (1938, pp. 3-25) :

- L'impulsion. Un problème survient qui inhibe ou arrête la poursuite d'une forme habituelle de conduite. Le problème indique l'écart entre ce que l'acteur anticipe dans la réponse de son geste et ce dont il fait effectivement l'expérience.

- La perception. Les perceptions de cet écart sont examinées afin de développer un diagnostic plus sophistiqué du problème et de ses causes. Cette analyse met à jour les conditions qui doivent être remplies avant que l'action puisse être poursuivie.

- La manipulation. Des hypothèses alternatives destinées à corriger le problème sont formulées et évaluées. À ce stade, la pensée de Mead est très proche de celle de Peirce (1903[1998]) et de sa notion de raisonnement abductif au moyen duquel des solutions créatives peuvent être générées.

- La consommation. Le problème est traité, en tout cas dans le moment présent, et l'activité continue bien que différemment que dans le schéma de conduite initial. L'effectivité de ce changement, bien que léger, est confirmée par les actions qui s'ensuivent et qui seront, en retour, sujettes à une analyse réflexive complémentaire lorsque les prochains problèmes apparaîtront.

Ce processus, par étapes, de pensée réflexive fait montre d'un fort parallèle avec la notion d'Inquiry de Dewey (1925[1998]) qu'il décrit en termes de cinq phases de pensée réflexive et d'action. Dans ces deux modèles de l'activité humaine en temps réel, les étapes ou les phases sont dynamiquement interdépendantes. Comme Miettinen (2000) le souligne, cette caractéristique distingue l'approche pragmatiste d'autres approches, telle celle de Kolb 
(1984) qui propose un modèle de l'apprentissage expérientiel qui est plus une typologie de différents modes d'apprentissage qu'un processus d'apprentissage dynamique.

La vision de la temporalité de Mead est qu'à la fois le passé et le futur sont dans les actions du présent. Le passé est composé de la multiplicité des attitudes sociales qui ont été constituées comme des symboles signifiants dans une situation sociale donnée alors que les futurs alternatifs sont abductivement anticipés et enactés. Bien entendu la nécessité de lier passé, présent et futur est bien acceptée par les théoriciens du processus (Pettigrew, 1990 ; Van de Ven \& Huber, 1990), mais la particularité de la vision de Mead vient de la façon dont il entrelace l'agentivité sociale avec cette dynamique temporelle. Les acteurs, situés entre le passé et le futur, sont contraints de reconstruire continûment leurs histoires dans le but de comprendre leurs transactions présentes. Dans le même temps, ils projettent ces compréhensions dans le futur pour en déduire les résultats probables de actions présentes.

C'est le 'je' qui est au cœur du principe dynamique de la théorie de Mead en tant qu'il enacte performativement le futur. Quand le 'je' se projette dans le futur, il est informé par les habitudes incarnées de conduite du 'moi'. Ainsi, le 'je' est la source d'alternatives créatives pour l'action qui justifient les possibilités émergentes dans les transactions. Cette notion d'émergence représente un défi pour les hypothèses téléologiques conventionnelles, car si le futur est déjà déterminé alors il n'y a pas de place pour la nouveauté et l'action créatrice. De ce fait, Mead plaide pour une téléologie non-intentionnelle dans laquelle la fin et les moyens des actions sociales sont co-constituées et co-évolutives, dans des contextes sociaux qui sont eux-mêmes en perpétuel changement. Par conséquent, il considère les actions sociales comme faiblement guidées par des desseins et des plans délibérés.

\section{Qu'est-ce que tout cela signifie pour la pratique ?}

Pour les pragmatistes, vivre implique un engagement actif et réflexif dans les transactions qui constituent l'expérience. Ici le terme 'expérience' a un sens très précis et particulier (Bernstein, 1972) référant aux aspects transactionnels, sociaux, réflexifs, projectifs et anticipatoires que j'ai mis en avant dans les lignes précédentes. La pratique est par conséquent la conduite d'une vie transactionnelle impliquant l'intrication temporelle et symboliquement médiatisée de l'expérience et de l'action. Cette définition de la pratique renvoie à un processus dynamique et émergent qui permet aussi bien des routines que des possibilités d'actions créatrices. Une implication clé de tout ceci est que les résultats de la pratique ne peuvent être prédits dans un sens téléologique. Ils sont plutôt des enactions émergentes dans un futur alors que les acteurs anticipent les suites probables de leurs actions sociales. Ces actes anticipatoires modèlent les choix des acteurs eu égard à leur conduite en devenir et, au bout du compte, modèlent également leurs mondes.

Une seconde implication clé de cette perspective pragmatiste est que la pratique et l'identité sont des processus co-constitués. Ce qui signifie que les acteurs tirent la signification et le sens de leur soi de leurs engagements transactionnels; leurs 'moi's sont continûment en devenir alors que la signification de leur conduite prend corps pour eux au sein de leurs transactions. Mead soutient que nous sommes incités à nous engager dans des pratiques transactionnelles pour pouvoir nous comprendre nous-mêmes; c'est seulement à travers notre conduite sociale que la conscience de soi peut apparaître. Le processus de construction de l'identité est par conséquent intimement associé avec cette notion de pratique, mais cependant les tendances actuelles dans la littérature des sciences des organisations tendent à placer la pratique et l'identité dans des sous-domaines bien séparés. La perspective pragmatiste offre une approche potentiellement plus fructueuse qui voit la pratique et l'identité comme étant des processus co-constitués, transactionnels et producteurs de significations.

Une troisième implication est le fait que la pratique est à la fois convergente et divergente. 
La convergence vers les normes de la conduite sociale peut être expliquée en invoquant des 'symboles signifiants' qui sont incarnées dans le 'moi' et donne un moyen d'établir et de réguler des attentes sociales de la conduite à travers l' 'autrui généralisé'. Parallèlement, la divergence vers la nouveauté et les différences émergentes est le fait des qualités performatives du 'je' qui donnent lieu au principe créatif d'abduction. Joas (1996) note en particulier que les perspectives 'rationnelle' et 'normativement orientée' attribuent toutes les deux à l'action créatrice un statut d'externalité. On peut cependant soutenir que ces deux perspectives pourraient être envisagées comme des cas particuliers d'une théorie de l'action créatrice inspirée par le pragmatisme. En subsumant ces deux champs théoriques actuellement dominants, Joas a mis l'accent sur le fait que l'approche pragmatiste pourrait les englober.

Cet ensemble d'implications met bien en lumière les principales figures de l'approche pragmatiste de la pratique, mais comment cela peut-il informer le tournant pratique ? Est-ce que cela conduit à de nouvelles façons de voir ou est-ce que cela fait surgir de nouvelles et intéressantes questions? De mon point de vue, la focalisation sur les transactions comme lieux de la construction de significations fournit un moyen non dualiste de modélisation de la pratique privilégiant ni la structure ni l'agentivité. Dans le même temps, la dynamique anticipatoire qui lie dans l'instant présent passé et futur offre une conception de la temporalité qui n'est limitée ni au présent en temps réel ou au passé historique, ni à une séquence unitaire d'événements dans un temps spatialisé. Ces éléments théoriques fournissent une façon très différente d'envisager la pratique comme un processus dynamique de construction sociale de significations et, dans le même temps, suggèrent plusieurs types de questions empiriques qui peuvent motiver une recherche. Par exemple, comment la pratique est-elle constituée à travers la combinaison de l'expérience et de l'action ; comment l'identité façonne-t-elle la pratique, et réciproquement; et comment l'agentivité sociale s'exprime-t-elle dans les différents types de transactions? Pour prouver l'utilité potentielle de cette approche pragmatique de la pratique, je me tourne maintenant vers une illustration s'appuyant sur une étude de cas publiée, relative à une pratique de stratégie. Ce faisant, mon objectif n'est pas de critiquer le travail publié ni d'analyser à nouveau le matériau empirique (auquel de toute façon je n'ai pas accès), mais plutôt de mettre l'accent sur les différents types de questions qui peuvent motiver une enquête orientée par une démarche pragmatiste.

\section{Un épisode de mise en place d'une stratégie}

L'exemple que j'ai choisi est l'étude de cas présenté par Maitlis et Lawrence (2003) dans laquelle ils décrivent les processus stratégiques d'un orchestre symphonique britannique. J'ai fait ce choix, car les auteurs proposent une présentation richement détaillée d'une 'histoire décisionnelle' suivant un épisode de mise en place d'une stratégie (strategizing) de plus de deux ans. Cet épisode survient au moment où l'orchestre doit faire face à la nécessité de changements dont un ensemble de nouvelles nominations (chef exécutif, chef d'orchestre principal, directeur du marketing, directeur artistique) et à de nouveaux impératifs commerciaux pour justifier les financements par le conseil des arts (Arts Council). Les principales parties prenantes, dont le conseil de l'orchestre, l'équipe de management et les musiciens, s'accordent toutes sur le fait qu'il y a clairement un besoin de définition d'une nouvelle stratégie reflétant l'identité artistique de l'orchestre; la formulation de cette stratégie est d'ailleurs inscrite dans la définition de la mission du nouveau directeur artistique. Cela dit, au moment où l'étude a été terminée, il n'y avait toujours pas de stratégie artistique faisant consensus, financièrement fiable et formellement documentée. Maitlis et Lawrence interprètent ceci comme un échec de la mise en place de la stratégie qu'ils expliquent en termes de déficit de la «combinaison entre certains des éléments du discours organisationnel et des types spécifiques de comportements politiques » (2003, p. 109).

Les arguments que les auteurs avancent reflètent des hypothèses particulières relatives la 
nature de la mise en place d'une stratégie vue comme un processus unitaire, orientée par son but et rationnelle. Par exemple, l'évocation d'un 'échec' semble impliquer une évaluation du succès pré-déterminée vis-à-vis de laquelle les efforts de mise en place de la stratégie par l'orchestre sont comparés. De telles hypothèses auront inévitablement façonné à la fois les questions de recherche qui ont motivé l'enquête et l'interprétation des données. Cependant, si cet épisode de mise en place de la stratégie avait été étudié à partir d'une approche pragmatiste de la pratique, les hypothèses sous-tendant la recherche auraient été bien différentes. La téléologie non-intentionnelle de Mead aurait ainsi dirigé la recherche vers des issues non prévues plutôt que vers des résultats pré-déterminés. Tout en admettant que cette approche puisse être, à première vue, profondément insatisfaisante pour les tenants de la stratégie orientée par son but, puisque que selon elle, c'est le caractère imprévu et émergent des choses qui défait les meilleurs plans et ainsi, je considère néanmoins ces types de questions comme constituant un domaine de développement intéressant pour la littérature sur la stratégie.

Méthodologiquement, cette étude de cas s'appuie sur une grande variété de données qualitatives dont des observations et des minutes officielles de plus de soixante réunions des différents groupes de parties prenantes, quarante interviews complètement retranscrits, des notes de terrains relatives à de nombreuses discussions informelles et des documents d'archives provenant du domaine public et des ressources internes. Le traitement des données par les auteurs s'opère en trois étapes: une description fine de 1' histoire décisionnelle' appuyée sur la chronique de tous les événements qu'ils ont jugé pertinents ; la codification de cette histoire secondaire destinée à identifier deux grands thèmes exploratoires ; le développement itératif d'un cadre conceptuel expliquant les événements de cet épisode de mise en place d'une stratégie dans les termes de ces deux thèmes. Un effet de cette stratégie d'analyse est d'éloigner très rapidement les chercheurs des données réelles (et de la pratique) en adoptant une interprétation plus abstraite privilégiant leurs propres voix.

Le même ensemble de données pourrait être également interrogé à partir d'une perspective pragmatiste en se focalisant sur la nature transactionnelle de l'épisode de mise en place de la stratégie. Cette approche pourrait poser la question de savoir quelles transactions ont été plus ou moins influentes dans le processus de mise en place de la stratégie, et également comment ces transactions ont contribué à la construction en cours des significations dans cet épisode. L'observation des réunions peut fournir de nombreuses opportunités pour étudier les transactions interpersonnelles et pour identifier les habitudes de conduite des différents groupes de parties prenantes. Une analyse fine des transactions à ce niveau peut potentiellement révéler la dynamique de convergence vers des attitudes communément adoptées et de divergence vers de nouvelles alternatives. Les transactions entre les parties prenantes internes, mais aussi entre l'orchestre et les financeurs ou les spectateurs, pourraient être également productives en termes de perspectives analytiques de la pratique de mise en place de la stratégie. Maitlis et Lawrence mentionnent par exemple des conflits entre les visions des différents groupes de parties prenantes; un pragmatiste pourrait se demander comment ces conflits ont été gérés entre ces groupes et avec quelles conséquences pour la construction de significations dans l'organisation.

Les interviews et les conversations pourraient également constituer une voie empirique fructueuse pour accéder à la pratique. Ils fournissent une occasion d'explorer les symboles signifiants que les individus utilisent dans leurs efforts particuliers pour construire des significations. Cependant, puisque ces symboles sont révélés par les gestes qui comprennent les actions verbales, émotionnelles et physiques, les données collectées dans les interviews seraient plus exploitables si, au-delà des transcriptions, on pouvait inclure des enregistrements sonores et des notes de terrain. De plus, les interviews invitent à la réflexivité ; le fait de poser des questions est un geste qui peut interrompre le flux de la conduite de l'interviewé, déclenchant une occasion de réfléchir; dans la même manière, une réponse de l'interviewé peut également rompre la réflexion du questionneur. La réflexivité 
de ce processus, dans lequel l'interviewé et l'intervieweur co-construisent des significations, est rendue explicite dans l'approche pragmatiste de la pratique.

Le problème de l'identité artistique de l'orchestre est au cœur de cet épisode de mise en place d'une stratégie. Les musiciens ont, par exemple, observé que « les programmes de la saison [1998/99] ne faisaient pas preuve d'une réelle identité artistique à une époque où de nombreux orchestres présentaient des cycles [d'œuvres d'un compositeur donné] » (Maitlis \& Lawrence, 2003, p. 117). Cependant le modèle théorique proposé par les auteurs ne concerne pas explicitement ces questions identitaires ; il les incite plutôt à focaliser plus généralement sur les ressources discursives. L'approche pragmatiste suggérerait que si la mise en place d'une stratégie est conformée par la pratique, alors la mise en place de la stratégie et l'identité sont intimement reliées en tant que processus se constituant mutuellement. Ainsi, les acteurs ne font pas que construire la signification de leur environnement stratégique, mais ils élaborent, dans le même temps, leur compréhension de leurs soi. Cette perspective conduit à aborder les ensembles de données en se focalisant non seulement sur les significations situées, mais également sur la construction des soi sociaux en contexte.

Dans cette partie de l'article, j'ai indiqué ce que cette approche pragmatiste pourrait offrir pour améliorer notre compréhension de la pratique et, ainsi, pour contribuer aux débats en cours dans le tournant pratique. En restant très proches des données et des pratiques transactionnelles sur le lieu de la recherche, les questions intéressantes soulevées par cette approche tournent autour des liens entre pratique et identité et du 'comment' de l'action sociale plutôt que de son 'pourquoi'. Cela ne veut pas dire cependant que cette perspective pragmatique de la pratique ne s'intéresse pas aux résultats de la mise en place de la stratégie ; mais là où les théories 'rationnelle' et 'normativement orientée' se centrent sur les résultats attendus et intentionnés, l'approche pragmatiste est plutôt concernée par les issues émergentes. Il y a ici évidemment des parallèles avec les approches de la recherche ethnographique, mais la figure distinctive du pragmatisme est l'intégration de la transactionnalité et de la temporalité dans une description holiste de la pratique sociale.

\section{En se tournant vers la pratique}

L'objectif de cet article est de renforcer la voix de la philosophie pragmatiste dans les débats qui alimentent actuellement le tournant vers la pratique dans les sciences des organisations. J'ai soutenu en particulier que la description détaillée de l'action sociale que propose Mead, en termes d'expression de l'agentivité sociale temporellement émergente et fondée transactionnellement, offre un riche potentiel pour de nouvelles conceptualisations et compréhensions de ce que font effectivement les gens dans les organisations. Il y a bien sûr déjà des exemples de propositions théoriques pragmatistes dans la littérature sur l'organisation, mais elles s'appuient le plus souvent sur les travaux de John Dewey (Cohen, 2007 ; Elkjaer, 2004 ; Miettinen , 2006). Mead est nettement moins présent et lorsqu'il est cité c'est le plus souvent sous forme d'une simple note relative à l'interactionnisme symbolique $^{2}$. Toute analyse approfondie de ses travaux est remarquablement absente. Une rare exception à cet état de fait est le travail de Hatch et Schultz (2002) qui tentent de travailler sur le 'moi' et le 'je' de Mead. Cela dit, les auteurs situent fautivement les idées de Mead à un niveau d'analyse individuel, manquant l'idée cruciale selon laquelle le 'moi' et le 'je' sont des aspects d'un soi irrémédiablement social. La première contribution que ce travail apporte cependant consiste à revisiter les idées de Mead en mettant à jour leur potentiel pour constituer une source de nouvelles perspectives pour la conduite humaine et la

2 Une analyse des citations de Mead dans vingt-deux journaux de haut niveau dans les domaines de l'organisation et du management montre que, dans les dix dernières années, $97 \%$ des citations renvoyaient à son livre de 1934 et n'offraient pas de perspectives critiques. 
construction de significations.

Deuxièmement, d'un point de vue théorique, une approche pragmatiste offre de nouvelles façons d'aborder la pratique. En particulier, elle lie explicitement la pratique et l'identité en tant que processus sociaux qui se constituent mutuellement. Emirbayer et Mische (1998, p. 992) observent que, depuis les années soixante-dix, les théories ont séparé artificiellement 'identité' et 'stratégie' et que ceci «a eu pour effet de rompre les liens entre les deux dimensions intrinsèquement liées de la mise en projet (projectivity) : les stratégies ont été dépossédées de la signification et de la réflexivité alors que les identités ont été temporellement aplanies et dépouillées de leur puissance d'orientation » (strategies are stripped of meaning and reflexivity, while identities are temporally flattened out and shorn of their orienting power). Je propose donc que ces théories des pratiques organisationnelles, telle la mise en place d'une stratégie, puissent être améliorées par la restauration de ce lien vital à l'identité.

L'approche pragmatiste questionne également directement la question théorique des dualismes. L'étude que Schatzki (2001) a consacrée aux théories contemporaines de la pratique, en termes de 'pratiques et ordre social', 'pratiques internes' et 'défis posthumanistes', révèle deux dualismes omniprésents, à savoir la séparation entre les niveaux d'analyse individuel et social, et celle posée entre l'action usuelle et l'action créatrice. La définition que Mead donne de la pratique transcende ces deux dualismes. Le soi social est vu comme émergeant continûment des transactions, émergence qui abolit la rupture individuel/social, et ce soi est conçu comme le fait d'une action ininterrompue, à la fois convergente et divergente, mais jamais statique. Même les vieilles traditions sont, par conséquent, sujettes à des reconfigurations à tout moment (Simpson and Hibbert, 2008). Ainsi la théorisation de Mead, se focalisant sur l' 'acte social' plutôt que sur les acteurs et les événements, élimine ces distinctions dualistes qui continuent à caractériser la plupart de la littérature contemporaine sur la pratique.

Troisièmement, le pragmatisme se particularise aux plans ontologique, épistémologique et méthodologique, offrant une perspective alternative dans les débats sur le tournant pratique. L'unité ontologique fondamentale dans cette approche est la transaction qu'elle soit intra-, inter- ou extra-personnelle. En réalité toute transaction offre une perspective sur la pratique pourvu que le cœur de l'enquête concerne la dynamique temporelle et agentique de la construction de significations plutôt que les significations spécifiques et les constructeurs de significations particuliers. Cette vision transactionnelle renvoie à une autre ontologie relationnelle. Par exemple la 'pensée-avec' de Shotter touche au caractère relationnel de nos transactions de construction de significations. Selon lui, les moments de réflexion «nous deviennent disponibles à partir de la dynamique révélée » par les personnes avec lesquelles nous sommes engagés dans une relation (Shotter, 2006, p. 599).

Épistémologiquement, le pragmatisme s'oppose à ce que Dewey appelle la théorie de la connaissance du 'spectateur' qui sépare l'observateur de l'observé (Dewey, 1917[1980]). Nous sommes des participants à des mondes que nous apprenons à connaître à travers nos actions sociales. Le fait de savoir n'est pas premier par rapport au fait d'agir ; les deux sont inextricablement liés dans la dynamique de la conduite humaine. Cette épistémologie participative trouve un écho dans la vision de la nature émergente et performative de la recherche scientifique que défend Pickering face à la vision plus conventionnelle selon laquelle le but de la science est de capturer et représenter fidèlement le monde dans lequel on vit. Son crédo performatif «part de l'idée selon laquelle le monde est rempli, en première instance, non pas de faits et d'observations, mais d'agentivité » (Pickering, 1995, p. 6).

Méthodologiquement, le pragmatisme met en avant une approche différente pour considérer la pratique, et dans le même temps ouvre de nouvelles et intéressantes questions à explorer. Il propose en particulier une voie nouvelle pour aborder le 'comment' la pratique émerge dans le temps réel plutôt que le 'pourquoi' la pratique est en œuvre. L'ethnométhodologie 
(Garfinkel, 1967) est également concernée avec les pratiques de tous les jours, en temps réel, dans les situations sociales (voir par exemple Samra-Fredericks, 2003, 2004), mais, dans son cas, le but de l'étude est de découvrir les sources de la régulation et de l'ordre social. Il y a ainsi dans les études ethnométhodologiques une intention propre de recherche de stabilité qui laisse peu de place pour comprendre les propriétés réflexives et créatrices de la pratique divergente (Miettinen, 2006).

Pour finir, je dois dire qu'en écrivant cet article, j'ai souvent choisi le domaine du management stratégique pour développer mon argument. Je ne souhaite cependant pas donner l'impression que c'est là le seul sous-domaine des sciences des organisations qui puisse bénéficier de cette approche pragmatiste. Je pense qu'elle peut s'appliquer dans tout domaine de pratiques que cela soit le savoir et l'apprentissage, l'innovation, la gestion du changement ou encore la myriade des pratiques constituant l'expérience organisationnelle. Il est intéressant de noter que cette théorie s'applique également à nos propres pratiques de chercheurs et, de ce fait, nous fournit des modalités très utiles pour réfléchir à nos activités scientifiques. Elle attire à l'évidence notre attention sur notre rôle en tant qu'acteurs et sur l'inévitable influence de notre agentivité sur les lieux de notre recherche. Dans le même temps, nos identités de chercheurs sont façonnées par notre engagement transactionnel avec les autres acteurs.

La position pragmatiste que j'ai présentée ici présente un grand intérêt, car elle offre une alternative aboutie aux philosophies représentationnelles qui tendent à dominer la réflexion sur la pratique. Je ne prétends cependant pas que le pragmatisme est une panacée ; je propose simplement une autre voie dans les débats en cours. Si nous sommes en marche pour réaliser pleinement le tournant de la pratique dans les sciences des organisations, c'est maintenant le moment d'élever le niveau du débat. Via la pratique transactionnelle du débat, on peut espérer éventuellement atteindre la précision et la réflexivité que le tournant de la pratique devrait comporter. Il y a encore beaucoup de travail à faire.

\section{NOTES}

Ce travail est le résultat émergeant (transactionnellement) d'un ensemble important de conversations avec beaucoup de personnes différentes. Je suis reconnaissante à tous ceux et toutes celles qui ont contribué à ce processus, mais je veux remercier particulièrement Gerry Johnson et Bente Elkjaer, chacun ayant parcouru avec moi un bout de chemin, et également John Sillince et Lee Edwards dont les retours critiques provocateurs ont fait avancer ma pensée. Je suis aussi redevable aux quatre relecteurs anonymes et aux trois éditeurs qui ont tous contribué à la forme finale de ce manuscrit.

\section{BIBLIOGRAPHIE}

Aboulafia, M. (1999). A (neo) American in Paris: Bourdieu, Mead, and Pragmatism. In R.

Shusterman (Ed.), Bourdieu: A critical reader (pp. 153-174). Oxford: Blackwell.

Bergson, H. (1919). Creative evolution. London: Macmillan.

Bernstein, R.J. (1972). Praxis and action. London: Duckworth.

Blackler, F., Norman C., \& McDonald, S. (2000). Organizing processes in complex activity networks. Organization, 7/2, 277-300.

Blumer, H. (1969). Symbolic interactionnism: Perpsective and method. London: University of California Press.

Burkitt, I. (1993). Social selves: Theories of the social formation of personality. London: Sage.

Callon, M. (1986). Some elements of a sociology of translation: Domestication of the scallops and fishermen of St. Brieuc Bay. In J. Law (Ed.). Power, action and belief: A new sociology of knowledge? (pp. 196-233). London: Routledge,

Chia, R., \& Holt, R. (2006). Strategy as practical coping: A Heideggerian perspective. Organization Studies, 27/5, 635-655.

Chia, R., \& MacKay, B. (2007). Post-processual challenges for the emerging strategy-as-practice perspective: Discovering strategy in the logic of practice. Human Relations, 60/1, 217-242. 
Cohen, M.D. (2007). Reading Dewey: Reflections on the study of routine, Organization Studies, 28/5, 773-786.

Cook, G.A. (1993). George Herbert Mead: The making of a social pragmatist. Urbana and Chicago: University of Illinois Press.

Cook, S., \& Yanow, D. (1993). Culture and organizational Learning. Journal of Management Inquiry, 2/4, 373-390.

Dewey, J. (1917 [1980]). Learning to earn: The place of a vocational education in a comprehensive scheme of public éducation. In J.A. Boydston (Ed.). The middle works, 1899-1924, Vol. 10. Carbondale and Edwardsville: Southern Illinois University Press.

Dewey, J. (1925 [1988]). Experience and nature. In J.A. Boydston (Ed.). The later Works, 1925-1953, Vol. 1. Carbondale and Edwardsville: Southern Illinois University Press.

Dewey, J., \& Bentley, A.F. (1949 [1991]). Knowing and the known. In J.A. Boydston (Ed.). The later Works, 1925-1953, Vol. 16. (pp. 1-294). Carbondale and Edwardsville: Southern Illinois University Press,.

Dunn, R.G. (1997). Self, identity and difference: Mead and the poststructuralists, Sociological Quarterly, 38/4, 687-705.

Elkjaer, B. (2004). Organizational learning: The "third way", Management Learning, 35/4, 419-434.

Emirbayer, M., \& Mische, A. (1998). What is agency? American Journal of Sociology, 103/4, 9621023.

Engeström, Y., Puonti, A. \& , Seppänen, L. (2003). Spatial and temporal expansion of the object as a challenge for reorganizing work. In D. Nicolini, S. Gherardi, \& D. Yanow (Eds.). Knowing in organizations (pp. 151-186). London and Armonk, NY: M. E. Sharpe,.

Ezzamel, M., \& Willmott, H. (2004). Rethinking strategy: Contemporary perspectives and debates. European Management Review, 1/1, 43-48.

Garfinkel, H. (1967). Studies in ethnomethodology. Englewood Cliffs, NJ: Prentice-Hall.

Harvey, D., \& Haraway, D. (1985). Trespassing on the future: A debate and discussion with David Harvey and Donna Haraway. Society \& Space, 13, 507-527.

Hatch, M.J., \& Schultz, M. (2002). The dynamics of organizational identity. Human Relations, 55/8, 989-1018.

Jarzabkowski, P. (2003). Strategic practices: An activity theory perspective on continuity and change. Journal of Management Studies, 40/1, 23-56.

Jarzabkowski, P. (2004). Strategy as practice: Recursiveness, adaptation, and practices-in-use. Organization Studies, 25/4, 529-560.

Jarzabkowski, P., Balogun, J., \& Seidl, D. (2007). Strategizing: The challenges of a practice perspective, Human Relations 60/1, 5-27.

Joas, H. (1996). Creativity of action. Chicago: University of Chicago Press.

Joas, H. (1997). G. H. Mead: A contemporary re-examination of his thought. Cambridge, MA: MIT Press.

Johnson, G., Melin, L., \& Whittington, R. (2003). Micro strategy and strategizing: Towards an activity-based view. Journal of Management Studies, 40/1, 3-22.

Kärreman, D., \& Alvesson, M. (2001). Making newsmakers: Conversational identity at work. Organization Studies, 22/1, 59-89.

Kilpinen, E. (1998). Creativity is coming. Acta Sociologica, 41, 173-179.

Knights, D., \& Mueller, F. (2004). Strategy as a 'Project': Overcoming dualisms in the strategy debate. European Management Review, 1/1, 55-61.

Knorr Cetina, K. (2001). Objectual practice. IN T.R. Schatzki, K. Knorr Cetina, \& E. Von Savigny (Eds.). The practice turn in contemporary theory (pp. 175-188). London and New York: Routledge.

Kolb, D. (1984). Experiential learning: Experience as the source of learning and development. Englewood Cliffs, NJ: Prentice-Hall.

Kuhn, T.S. (1975). The structure of scientific revolutions. Chicago: University of Chicago Press.

Latour, B. (1987). Science in action: How to follow scientists and engineers through society. 
Cambridge, MA: Harvard University Press.

Lave, J., \& Wenger, E. (1991). Situated learning: Legitimate peripheral participation. New York: Cambridge University Press.

Linstead, S. (2002). Organization as reply: Henri Bergson and casual organization theory. Organization, 9/1, 95-111.

Maitlis, S., \& Lawrence, T.B. (2003). Orchestral manoeuvres in the dark: Understanding failures in organizational strategizing. Journal of Management Studies, 40/1, 109-139.

Mead, G.H. (1925). The genesis of the self and social control, International Journal of Ethics. 35, 251-77.

Mead, G.H. (1932). The philosophy of the present. Illinois: La Salle.

Mead, G.H. (1934). Mind, self and society. Chicago: University of Chicago Press.

Mead, G.H. (1938). The philosophy of the act. Chicago: University of Chicago Press.

Mead, G.H. (1963-4). Relative space-time and simultaneity. Review of Metaphysics, 17, 514-535.

Miettinen, R. (1999). The riddle of things: Activity theory and actor-network theory as approaches to studying innovations. Mind, Culture and Activity, 6/3, 170-195.

Miettinen, R. (2000). The concept of experiential learning and John Dewey's theory of reflective thought and action. International Journal of Lifelong Education, 19/1, 54-72.

Miettinen, R ; (2006). Epistemology of transformative material activity: John Dewey's pragmatism and cultural-historical activity theory. Journal for the Theory of Social Behavior, 36/4, 389-408.

Morris, C.W. (1934). Introduction: George H. Mead as social psychologist and social philosopher. In C. W. Morris (Ed.) Mind, self, and society from the standpoint of a social behaviorist. Chicago and London: University of Chicago Press.

Nicolini, D., Gherardi, S., \& Yanow, D. (2003). Knowing in organizations: A practice-based approach. London and New York: M. E. Sharpe.

Orlikowski, W.J. (2000). Using technology and constituting structures: A practice lens for studying technology in organizations. Organization Science, 11/4, 404-428.

Peirce, C.S. (1903[1998]). The essential Peirce. Bloomington: Indiana University Press.

Pettigrew, A.M. (1990). Longitudinal field research on change: Theory and practice. Organization Science, 1/3, 267-292.

Pettigrew, A.M. (2008). Re-viewing 'role' in processes of identity construction. Organization, 15/1, 29-50.

Pickering, A. (1995). The mangle of practice: Time, agency and science. Chicago and London: University of Chicago Press.

Powell, T.C. (2002). The philosophy of strategy. Strategic Management Journal, 23/9, 873-880.

Reckwitz, A. (2002). Toward a theory of social practices: A development in culturalist theorizing. European Journal of Social Theory, 5/2, 243-263.

Rescher, N. (2000). Process philosophy: A survey of basic issues. Pittsburgh: University of Pittsburgh Press.

Rorty, R. (1999). Philosophy and social hope. London: Penguin.

Samra-Fredericks, D. (2003). Strategizing as lived expérience and strategists' everyday efforts to shape strategic direction. Journal of Management Studies, 40/1, 141-174.

Samra-Fredericks, D. (2004). Managerial elites making rhetorical and linguistic 'moves' for a moving (emotional) display. Human Relations, 57/9, 1103-1143.

Schatzki, T.R. (2001). Introduction: Practice theory. In T.R. Schatzki, K. Knorr Cetina, \& E. Von Savigny (Eds.). The practice turn in contemporary theory (pp. 1-14). London and New York: Routledge.

Shotter, J. (2006). Understanding process from within: An argument for 'withness'- thinking. Organization Studies, 27/4, 585-604.

Simpson, B., \& Carroll, B. (2003). Longitudinal field research on change: Theory and practice. Organization Science, 1/3, 267-292.

Simpson, B., \& Hibbert, P. (2008). Identity change in the context of long-established traditions. 
International Journal of Public Administration, 31/9, 1110-1126.

Tsoukas, H., \& Chia, R. (2002). On organizational becoming: Rethinking organizational change. Organization Science, 13/5, 567-582.

Van de Ven, A.H., \& Huber, G.R. (1990). Longitudinal field research methods for studying processes of organizational change. Organization Science, 1/3, 213-219.

Wenger, E. (2000). Communities of practice and social learning systems. Organization Science, 7/2, $225-246$.

Whitehead, A.N. (1920). The concept of nature: Tarner lectures delivered in Trinity College, November 1919. Cambridge: The University Press.

Whittington, R. (2004). Strategy after modernism: Recovering practice. European Management Review, 1/1, 62-68.

Whittington, R. (2006). Completing the practice turn in strategy research. Organization Studies, 27/5, 613-634.

Yanow, D. (2000). Seeing organizational learning: A 'cultural' view. Organization, 7/2, 247-268.

\section{RESUME}

Le terme 'pratique' est courant dans le langage de tous les jours, mais possède aussi une longue histoire dans le monde académique. Que veut donc dire se 'tourner' vers la pratique et comment peuton savoir quand un tournant pratique a eu lieu ? Pour répondre à ces questions, l'article propose une vision théorique de la pratique comme un processus social transactionnel impliquant l'action et l'expérience comme des aspects mutuellement informés de la conduite humaine. Cette perspective est élaborée en détail en exploitant les idées des philosophes pragmatistes, et spécialement celles de George Herbert Mead. On avancera en particulier que la 'transactionalité' et la 'temporalité', prises ensemble, offrent une perspective théorique sur la pratique qui est dynamique, émergente et socialement agentique. L'utilité de cette approche pragmatiste est illustrée à travers l'usage d'une étude publiée, relative à un épisode de mise en place d'une stratégie. L'article conclut qu'un tournant pratique est en cours dans les sciences des organisations, mais qu'il y a encore une bonne distance à parcourir avant que le potentiel de ce tournant soit actualisé.

\section{MOTS CLES}

Émergence, pragmatisme, agentivité sociale, temporalité.

\section{REFERENCEMENT}

Simpson, B. (2013). Le pragmatisme, Mead et le tournant pratique. Activités, 10(1), 155-171, http://www .activites.org/v10n1/v10n1.pdf

Article soumis le 8 septembre 2012, accepté pour publication le 16 février 2013. 